\title{
La reconstruction des ingénieurs 1942-1958
}

Engineers' Reconstruction (1942-1958)

\section{Nicolas Nogue}

\section{OpenEdition}

Journals

Édition électronique

URL : http://journals.openedition.org/crau/448

DOI : $10.4000 /$ crau. 448

ISSN : 2547-5746

Éditeur

Éditions du patrimoine

Édition imprimée

Date de publication : 1 mars 2014

Pagination : $37-54$

ISBN : 978-2-7577-0359-5

ISSN : 1296-4077

Référence électronique

Nicolas Nogue, "La reconstruction des ingénieurs 1942-1958 », Les Cahiers de la recherche

architecturale et urbaine [En ligne], 29 | 2014, mis en ligne le 13 septembre 2017, consulté le 02 mai

2019. URL : http://journals.openedition.org/crau/448; DOI : 10.4000/crau.448 
La période de la Reconstruction a produit une historiographie surtout axée sur l'intervention des architectes dans le domaine de l'architecture et de I'urbanisme, alors que les ingénieurs ont aussi joué un rôle déterminant.

Est ici soulignée la diversité des statuts professionnels et des modes d'exercice L'article montre ensuite que l'œuvre des ingénieurs est directement déterminée par les lignes de force qui régissent l'ensemble de l'économie française : la planification, le modèle de l'économie concertée, les priorités de la Reconstruction liées aux aides financières du plan Marshall. Les ingénieurs se sont engagés dans l'édification et la modernisation des équipements productifs fondamentaux pour la reprise économique du pays. Inscrits dans la problématique de l'aménagement du territoire, ces équipements se composent des grandes infrastructures liées au transport et à la production d'énergie.

\section{La Reconstruction des ingénieurs 1942-1958}

\section{L'aménagement de la productivité du territoire}

Quel rôle jouèrent les ingénieurs dans la reconstruction du pays dévasté ? Répondre précisément à ces questions relève de la gageure dans la mesure où les principales recherches historiques publiées à ce jour n'ont pas étudié spécifiquement l'ensemble de la contribution des acteurs de l'ingénierie après le conflit mondial ${ }^{1}$. Elles se sont surtout centrées sur la reconstruction urbaine où domina, en grande partie dans le discours mais aussi dans la pratique, l'intervention des architectes qui s'en trouve du même coup valorisée.

Il est vrai qu'une vision globale de l'activité des ingénieurs s'avère particulièrement difficile à préciser en raison, principalement, de leur statut et de leur exercice professionnel. Alors que les architectes exercent, dans leur immense majorité, en tant que maîtres d'œuvre libéraux, les ingénieurs offrent une palette d'activités beaucoup plus large. Ils sont fonctionnaires - notamment pour les ingénieurs des Ponts et Chaussées -,

1. On pense en particulier aux travaux d'Anatole Kopp, de Danièle Voldman et de Bruno Vayssière. Voir Anatole Kopp, Frédérique Boucher, Danièle Pauly, L'Architecture de la Reconstruction en France, 1945-1953, Paris, Le Moniteur, 1982 ; Bruno Vayssière, Reconstructiondéconstruction. Le "hard French" ou l'architecture française des Trente Glorieuses, Paris, Picard, 1988 ;

Reconstructions et modernisations.
La France après les ruines 1918-1945, Paris, Archives nationales, 1991 : Danièle Voldman, La Reconstruction des villes françaises de 1940 à 1954. Histoire d'une politique, Paris, L'Harmattan, 1997. 
en administration centrale et dans les collectivités territoriales; et comme les architectes, il exercent également en tant que libéraux au titre d'ingénieursconseils ou encore en tant que salariés d'entreprises, à l'instar de l'une des grandes figures de l'ingénierie française du moment : Nicolas Esquillan (1902-1989), directeur technique des établissements Boussiron. Les ingénieurs ont la possibilité d'exercer leur activité dans le cadre de la maîtrise d'œuvre mais aussi de la maîtrise d'ouvrage (voire en cumulant les deux fonctions). C'est le cas en particulier des ingénieurs des Ponts et Chaussées, des hauts fonctionnaires des administrations centrales parisiennes ou des dirigeants des grandes entreprises publiques - en particulier la SNCF, EDF, GDF et les Charbonnages de France - qui ont joué un rôle prépondérant dans la Reconstruction. Citons : Paul Peirani et Roger Vallette, respectivement chefs des divisions bâtiments et ouvrages d'art de la SNCF, Pierre Simon, premier P-DG d'Électricité de France ou encore Pierre Massé et Raymond Giguet qui se sont succédé à la direction de l'équipement d'EDF après $1945^{2}$. Sous la conduite de Massé qui s'inspire des méthodes des planificateurs, l'ensemble du programme de production électrique - hydraulique et thermique - a été conçu et suivi à l'aune de modélisations économétriques les plus sophistiquées du moment en France. Il s'agit d'optimiser sur l'ensemble du territoire national le choix du type d'électricité à produire, les montants et la localisation des investissements à opérer, les coûts d'entretien, de production et de transport afin de répondre, sans rupture d'approvisionnement, aux besoins extrêmement fluctuants de l'industrie privée et du secteur public. Cette méthode économétrique a été adoptée plus tard, en 1969, à I'heure de la rationalisation des choix budgétaires par l'administration centrale ${ }^{3}$.

Soulignons enfin le rôle des ingénieurs dans l'organisation générale du redressement du pays, que ce soit dans les principaux ministères reconstructeurs comme dans certains organismes parapublics qui, à l'instar de I'Afnor ou du CSTB, ont constitué des pièces maîtresses dans la politique d'industrialisation du bâtiment conduite par les pouvoirs publics ${ }^{4}$. Ainsi, même si I'on ne dispose pas d'études pointues à ce sujet, il est clair que les ingénieurs des Ponts et Chaussées ont occupé la totalité (ou presque) des postes de direction du ministère des Transports et des Travaux publics. La question mériterait par ailleurs d'être étudiée en ce qui concerne le ministère de la Production industrielle.

Au ministère de la Reconstruction et de l'Urbanisme (MRU), en collaboration avec les architectes, la contribution des ingénieurs s'avère essentielle, avec les figures de Raoul Dautry, premier dirigeant du MRU, de Jean Kérisel, directeur du Plan, d'Adrien Spinetta, directeur de la Construction qui a donné, à partir de 1950, une impulsion décisive à la politique des " grands ensembles ${ }^{5}$. Dans le cadre des instances de l'urbanisme, les ingénieurs des Ponts et Chaussées ont également occupé nombre de postes de commande. Dans l'ouvrage sur I'histoire du corps, André Brunot et Roger Coquand soulignent à ce propos que beaucoup de chefs des services des Ponts et Chaussées ont cumulé leur fonction avec celle de délégué départemental du MRU (on en comptait dix-huit en 1949 sur une soixantaine de délégations) ; en outre, Daniel Boutet, vice-président du conseil général
2. Pierre Massé occupa le poste de directeur de l'Équipement d'EDF de 1946 à 1948

Raymond Giguet de 1948 à 1955. Voir à ce propos, Dominique Barjot, Travaux Publics de France, un siècle d'entrepreneurs et d'entreprises (1883-1992), Paris, Presses de l'École nationale des ponts et chaussées, 1993, p. 145-163.

3. Voir Marc Desportes et Antoine Picon, De l'espace au territoire, L'aménagement en France $X V I^{e}-x x^{e}$ siècles, Paris, Presses de
I'École nationale des ponts et chaussées, 1997, p. 139.

4. Citons en particulier Albert Caquot, ancien ingénieur des Ponts et Chaussées, qui fut le président de l'Afnor à partir de 1943, et André Marini, directeur du CSTB à sa création, en 1947.

5. Ingénieur des Ponts et Chaussées, Adrien Spinetta (né en 1908) occupa au MRU le poste de directeur de la Construction de
1951 à 1956. II mit en place le « secteur industrialisé » qui, favorisant le lancement de grandes opérations de l'ordre de 1000 logements, est considéré comme l'une des étapes clés dans l'essor de la politique des grands ensembles. Voir Manuel Candré, Bruno Vayssière, Danièle Voldman, Une politique du logement. Ministère de la Reconstruction et de I'Urbanisme 1944-1954, Paris, Institut français d'architecture / Plan construction architecture, 1995, p. 96 et p. 139. 
des Ponts et Chaussées a présidé le Comité national d'urbanisme, chargé de l'examen au plus haut niveau des plans des cités à rebâtir ${ }^{6}$.

Intervenant à des postes clés de l'administration, de la conception et de la construction des grands équipements et de l'économie, œuvrant en tant que maîtres d'œuvre et maîtres d'ouvrage selon des fonctions et des statuts divers, les ingénieurs ont contribué à la Reconstruction selon des modalités à la fois aussi essentielles mais plus complexes et difficiles à saisir que celles empruntées par les architectes dont l'activité s'est surtout limitée au projet architectural et urbain.

On tentera d'en rendre compte, à grands traits, en concentrant surtout notre propos sur leurs activités de maître d'œuvre et par le biais de trois approches croisées : I'intervention du corps des Ponts et Chaussées tout d'abord, dans le domaine de l'urbanisme et des infrastructures; puis l'œuvre des grandes figures de l'ingénierie d'entreprise, leurs principales innovations techniques qui ont ouvert la voie au renouveau de l'art de bâtir des années 1950 ; enfin, leur contribution à l'un des mouvements majeurs de la période : I'industrialisation du bâtiment.

\section{L'urbanisme et les infrastructures \\ L'implication du corps des \\ Ponts et Chaussées}

Comme l'ont indiqué les spécialistes de la période, I'urbanisme des villes à reconstruire résulta d'une collaboration, plus ou moins heureuse selon les cas, entre architectes et ingénieurs des Ponts et Chaussées.
Toutefois, l'implication de ces derniers, bien qu'omniprésente, demeura relativement obscure tant ils occupèrent peu le champ médiatique et n'intervinrent pas dans les débats théoriques et doctrinaux où s'imposèrent en revanche les architectes ${ }^{7}$.

Dans la pratique, la domination apparente des architectes est à nuancer. Le corps des Ponts et Chaussées avait en effet obtenu depuis la fin du XIX ${ }^{\mathrm{e}}$ siècle une place accrue dans le domaine de l'urbanisme par le biais de la programmation des voiries et réseaux divers et, dès 1940, c'est-à-dire dès les premières destructions, ses services techniques avaient été sollicités sous l'autorité d'un Commissaire technique à la reconstruction immobilière qui était ingénieur des Ponts et Chaussées ${ }^{8}$. À la Libération ces prérogatives furent menacées par la politique du MRU, globalement favorable aux architectes en matière d'urbanisme : I'État avait non seulement opté pour le maintien de l'Ordre mais s'était aussi appuyé sur la nouvelle institution dans la procédure de recrutement des cadres opérationnels de la reconstruction urbaine, ce qui revenait à privilégier les architectes pour ces missions. C'est ainsi par l'intermédiaire du Conseil supérieur de l'Ordre que furent déposées au MRU, selon le vœu de Raoul Dautry, les candidatures aux postes d'urbanistes et d'architectes de la Reconstruction.

S'ils n'occupaient pas le terrain médiatique, les ingénieurs s'efforcèrent en revanche de préserver leurs prérogatives. Leur administration de tutelle, le ministère des Travaux publics, parvint ainsi le 30 avril 1947 à un accord avec le MRU leur garantissant une relative autonomie : ils conservaient la responsabilité des plans de voirie élaborés par l'urbaniste en chef et, en cas de modification
6. André Brunot, Roger Coquand, Le Corps des Ponts et Chaussées, Paris, Éditions du CNRS, 1982, p. 576-577. Sur I'organisation et les missions du Comité national d'urbanisme, CNU, voir plus particulièrement D. Voldman, op. cit. note 1 , p. 287-292.
7. Voir Gilles Jeannot, «Les ingénieurs dans la Reconstruction: images et stratégies», dans D. Voldman (dir.), Images, discours et enjeux de la reconstruction des villes françaises après 1945, Les Cahiers de l'IHTP, $n^{\circ}$ 5, Paris, CNRS, juin 1987, p. 69-82.
8. À propos du poids considérable du corps des Ponts et Chaussées dans les instances de l'urbanisme sous Vichy, voir Pierre Randet, "1941-1951. Panorama de la Reconstruction française ", dans B. Vayssière, Reconstructions et modernisations, op. cit. note 1, p. 95 
de ce dernier et de désaccord, pouvaient passer outre l'avis des délégations départementales du MRU pour en référer directement à l'administration centrale qui, soucieuse de ne pas ralentir les opérations, leur donna souvent raison. Mais, en général, les ingénieurs des Ponts et Chaussées surent collaborer sans trop de heurts avec les architectes qui dirigeaient l'élaboration des plans afin de définir rapidement la nouvelle implantation des logements, des voiries et des autres infrastructures de transport ${ }^{9}$.

Cela étant, une innovation majeure dans le domaine de l'urbanisme d'après-guerre fut introduite suivant les idées en matière de décongestion industrielle que l'ingénieur G. Dessus avait exprimées dès 1941 au sein de la délégation générale à l'Équipement national. L'intérêt donné progressivement aux initiatives des acteurs locaux et régionaux, la création - au sein du ministère de la Reconstruction et de l'Urbanisme - d'une direction de l'Aménagement du territoire prônant la politique élaborée par l'ingénieur P. Randet et, enfin, la fondation d'un Fonds national d'aménagement du territoire (FNAT) explique la naissance des nouvelles «zones industrielles » bâties en marge des cités $^{10}$. Au-delà, cette politique s'est développée à partir de 1951 pour permettre la réalisation de grands aménagements régionaux (réseaux d'eau, de routes, construction de barrage, de réseaux de distribution de l'électricité, construction de canaux) en liaison avec les bassins de main-d'œuvre urbaine. Comme le soulignent Marc Desportes et Antoine Picon qui se réfèrent aux propos de Claudius-Petit : « Le plan d'aménagement tente d'obtenir une meilleure localisation des activités et des hommes qui produisent ${ }^{11}$. »
Outre l'urbanisme, le rôle principal que jouèrent les ingénieurs des Ponts et Chaussées après 1945 s'illustra d'abord par la prise en charge, sur l'ensemble du territoire, des travaux de déblaiement et des constructions provisoires et, surtout, par la reconstruction des ouvrages d'art et grandes infrastructures de transport: ports maritimes et fluviaux, voies navigables intérieures, routes et ponts, aéroports civils et militaires. II s'agissait là d'un chantier gigantesque, organisé sous la houlette du ministère des Travaux publics et des Transports, pour lequel l'ingénierie civile et privée, intégrée aux entreprises ou indépendante (sous la forme des bureaux d'études techniques) fut largement sollicitée, ainsi que quelquefois les architectes $^{12}$

Ce champ d'intervention, avec ceux de l'industrie lourde (sidérurgie) et de l'énergie, étaient considérés comme prioritaires par les pouvoirs publics et, plus précisément, par le plan Monnet (1946-1952) qui favorisa particulièrement le BTP : parmi les six secteurs économiques à redresser d'urgence, le premier plan quinquennal de «modernisation et d'équipement de la France " en avait sélectionné cing concernant directement la construction ${ }^{13}$ : le charbon, l'électricité, I'acier, les transports et le ciment. Ils furent ainsi les premiers bénéficiaires de l'aide américaine du plan Marshall. Comme le souligne Danièle Voldman ${ }^{14}$ : «Pour les modernisateurs, les villes étaient des réservoirs de main-d'œuvre et la Reconstruction passait par un équipement général où les problèmes d'urbanisme ne jouaient qu'un rôle secondaire. Il leur semblait évident qu'on ne construirait pas de logements sans ciment et que celui-ci ne serait lui-même produit sans charbon ni électricité. » Les ordres
9. Selon André Brunot et Roger Coquant, « la collaboration des ingénieurs et des architectes s'imposait : elle fut généralement fructueuse, non seulement sur le terrain, mais par l'établissement, dans de nombreux cas, de relations professionnelles enrichissantes pour les deux parties (op. cit. note 6, p. 577). Plus circonspecte, Danièle Voldman précise qu'à part le cas exemplaire de collaboration étroite à Brest entre l'ingénieur Piquemal et I'urbaniste Mathon, « des difficultés entre urbanistes et ingénieurs n'ont pas manqué : les ingénieurs récusaient les arguments esthétiques des urbanistes au profit de solutions plus simples et moins coûteuses. Elles corroborent le cas général : au mieux une répartition sourcilleuse des tâches, au pire une collaboration méfiante » (op. cit. note 1, p. 263).

10. Le rapport qu'a rédigé Pierre Randet et qui fonde les principaux éléments de la politique d'aménagement du territoire présentés par Claudius-Petit en 1949 au Conseil des ministres est intitulé : Pour un Plan d'aménagement du territoire. Voir

P. Randet, L'Aménagement du territoire. Genèse et étapes d'un grand dessein, Paris, La Documentation française, 1994.

11. Op. cit. note 3, p. 146.

12. Pour les gares (maritimes, ferroviaires ou aériennes), il était le plus souvent fait appel aux architectes. Ils intervenaient 
de priorités ainsi définis se répercutaient directement sur les budgets des ministères concernés : entre 1946 et 1952, les crédits engagés par le MRU n'atteignaient que $10 \%$ de ceux du ministère des Travaux publics.

L'intervention majeure des ingénieurs des Ponts et Chaussées s'inscrivait donc dans le cadre prioritaire du redressement économique du pays. Intervenant dans le domaine de la construction, un autre groupe d'acteurs partageait le même objectif : les entreprises nationalisées comme la SNCF, EDF, GDF, la Compagnie nationale du Rhône et les Charbonnages de France où les ingénieurs (en particulier polytechniciens et ingénieurs des Ponts) occupaient également les principaux postes de direction ${ }^{15}$. Ces entreprises, on le verra, présidèrent à l'exécution des ouvrages aux technologies les plus avancées du moment, comme les barrages et les centrales électriques. À l'instar des programmes dirigés par le corps des Ponts et Chaussées, les grandes sociétés publiques de transport et d'énergie firent aussi souvent appel, pour la conception de leurs ouvrages, à l'ingénierie des bureaux d'études techniques (BET) et entreprises de construction (et à des architectes). Car, si les ingénieurs civils ne bénéficièrent pas de champs d'intervention privilégiés, comme ce fut schématiquement le cas pour les architectes et les ingénieurs d'État, ils figurèrent en revanche parmi les principaux acteurs de l'innovation technique.

\section{Ingénieurs, ouvrages et innovations techniques}

En raison de l'ampleur des chantiers ouverts, de la diversité des programme abordés, de la pénurie de main-d'œuvre et de matériaux à prendre en compte ainsi que des contraintes drastiques à respecter en termes de coût et de délai, la Reconstruction constitua un vaste laboratoire d'expérimentation technique, économique et esthétique qui influença profondément l'architecture contemporaine et où s'illustrèrent les grandes figures de l'ingénierie française du moment, en particulier dans le domaine des travaux publics ${ }^{16}$.

À cet égard, les ouvrages d'art donnèrent lieu à des innovations particulièrement fécondes, en termes d'organisation de la mise en œuvre, de préfabrication, et de nombreux records mondiaux de portée furent alors atteints ${ }^{17}$. Parmi les exemples innombrables, citons notamment la mise au point par les établissements Boussiron d'une nouvelle technique promise à un bel avenir, la construction en encorbellement à partir de chaque pile, pour le chantier du pont Raymond-Poincaré à Lyon, et, surtout, la diffusion, grâce aux travaux pionniers d'Eugène Freyssinet (1879-1962), de la précontrainte du béton, révolution technologique améliorant la performance des structures (portée, résistance, vieillissement...) tout en réduisant le coût de leur mise en œuvre ${ }^{18}$.

Ayant mûri ses conceptions depuis le début du siècle, I'ancien ingénieur des Ponts et Chaussées avait breveté l'invention en 1928 et commencé son application, dès le début des années 1930, par la construction de poteaux électriques préfabriqués puis d'autres ouvrages en France, en Allemagne et en Afrique du Nord ${ }^{19}$. En 1943, grâce à I'appui d'Edmé Campenon, il fondait son bureau d'études, la Société technique d'utilisation de la précontrainte (STUP), filiale de l'entreprise Campenon-Bernard, et marqua la Reconstruction française par l'exécution de nombreux ouvrage d'art. Le aussi parfois aux côtés des ingénieurs, en collaboration plus ou moins étroite, pour la conception d'ouvrages d'art, de barrages, de centrales électriques et autres grands équipement industriels et techniques, à l'instar de Théo Sardnal, un élève de Perret, pour la construction de I'usine André-Blondel à Bollène à partir de 1949. À ce titre, I'activité de l'architecte Jean Démaret est également exemplaire : il collabore avec Esquillan et Lossier pour la réalisation des ponts de Conflans-Fin-
d'Oise (1947-1949) et de Villeneuve-SaintGeorges (1948-1950). On le retrouve aux côtés de l'architecte Jean Fayeton et de I'ingénieur Robert Gibrat pour l'élaboration de la centrale thermique Émile-Huchet de Carling (1947-1953). Il est vrai que Démaret, comme Fayeton d'ailleurs, était également ingénieur centralien ce qui facilitait sans doute son intervention sur des ouvrages très techniques.
13. Voir D. Barjot « Les entreprises du bâtiment et des travaux publics et la Reconstruction (1918-1945), dans B. Vayssière, Reconstructions et modernisations, op. cit. note 1, p. 234.

14. Op. cit. note 1, p. 9.

15. A. Brunot et R. Coquand, op. cit. note 6, chapitre IV, "L'essaimage des ingénieurs des Ponts et Chaussées », p. 605-611. 


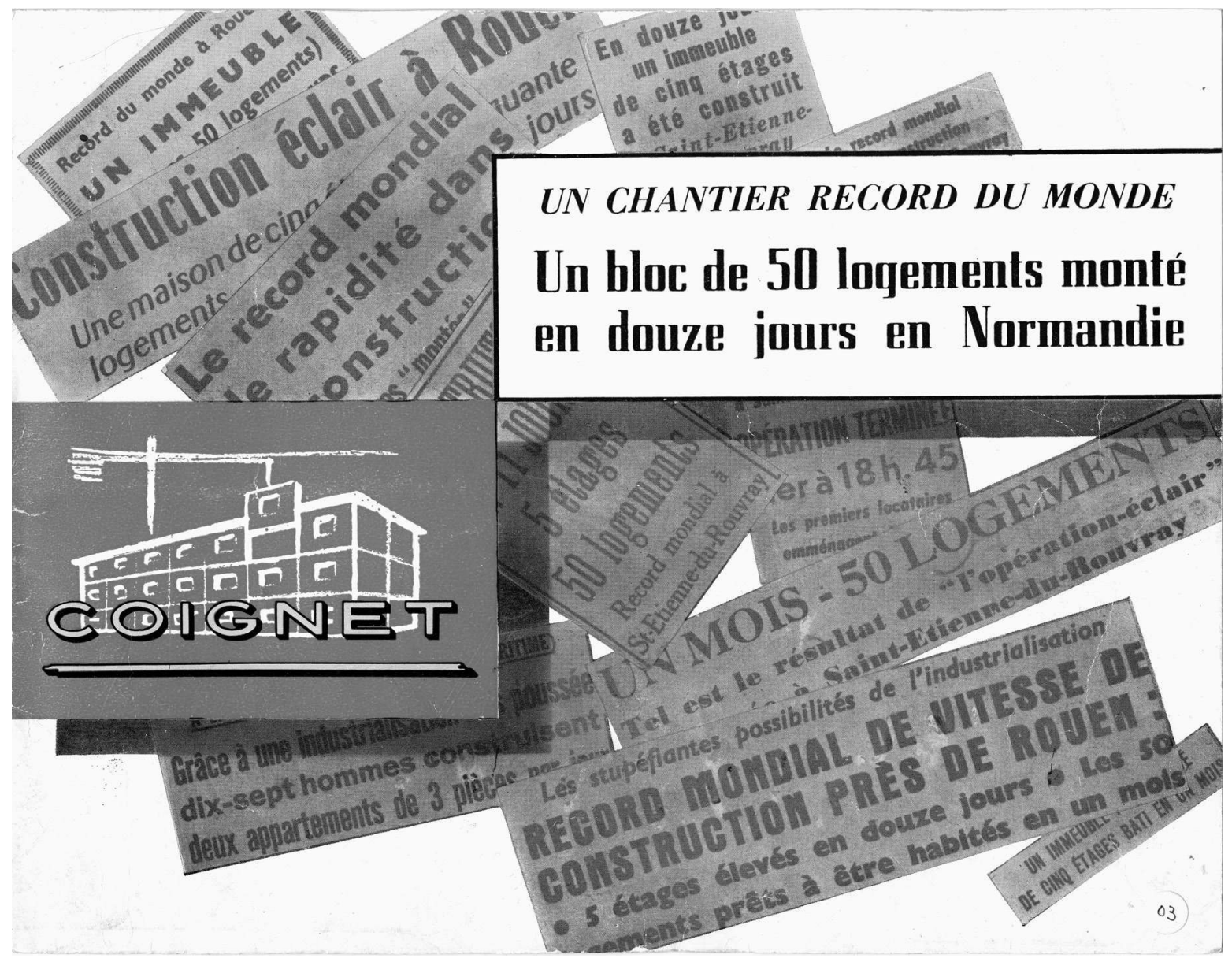

5 étages en 12 jours est-ce possible?
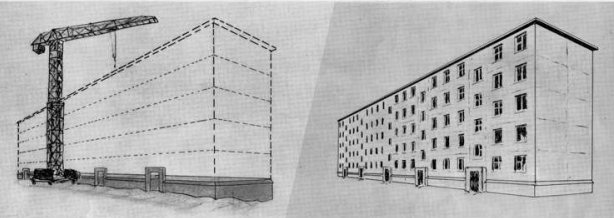

Elever en douze jours un immeuble de einq étages, le doter de tout le confort moderne et remettre les clés des einquante appartements terminés trente jours après la pose de la première pierre, tel est le record que voulait établir l'entreprise Edmond Coignet. L'industrialisation totale de la construetion devait permettre cette performanee. C'est ainsi qu'à Saint-Etienne-du-Rouvray, près de Rouen, fut lancé ce que la presse a appelé l'Opération éclair. Et voici ce qu'il en est advenu... 


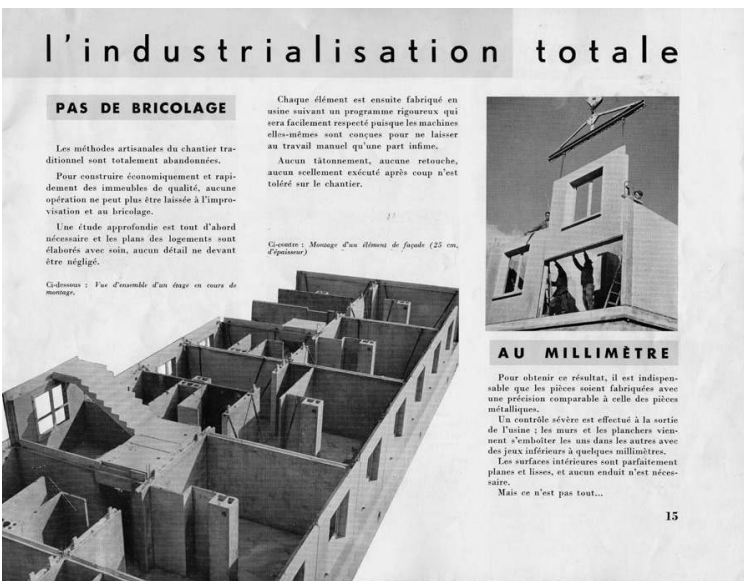

Extraits d'une

brochure de publicité

"Opération éclair ",

de l'entreprise

Coignet, 1958.

Construction de

50 logements en

12 jours à Rouen

à l'aide des procédés de préfabrication et

du mode de

production industria-

lisé de l'entreprise.

D.R

malgré les intempéries

17 hommes tiennent le délai
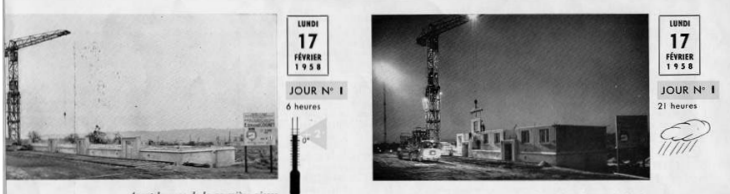

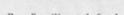
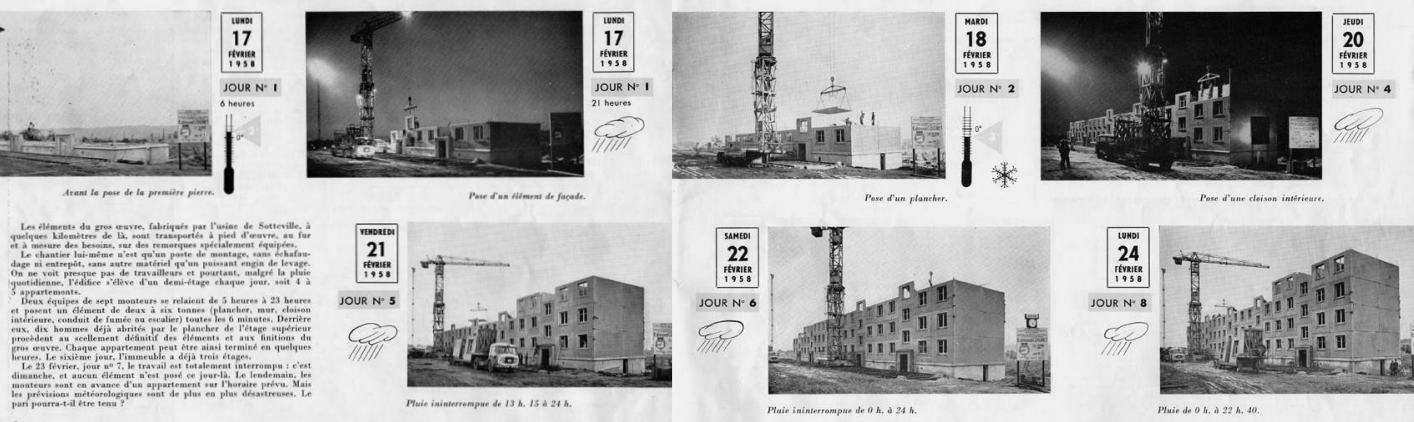

\section{2 jours de montage et la}
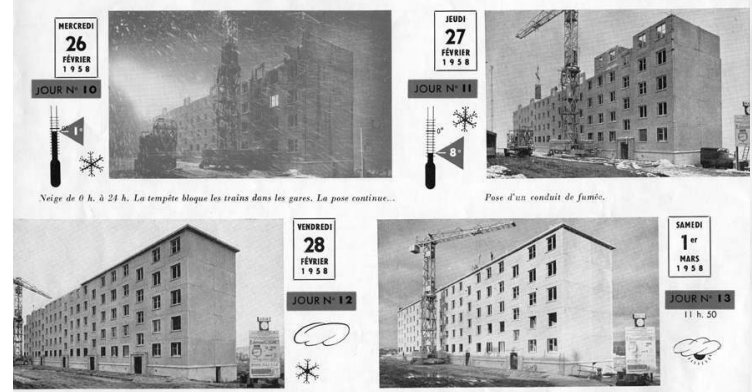

$d$ e $r n$ i è $r$ e pie $r r e$ e s t pos é e
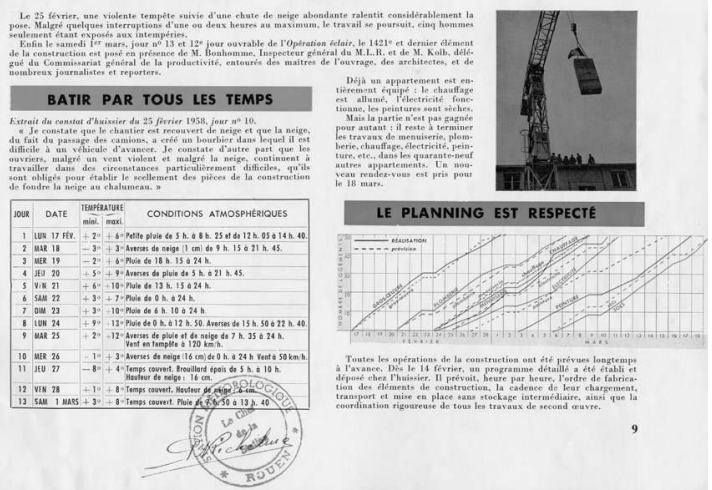
premier d'entre eux, achevé en 1945 à Luzancy sur la Marne, s'imposa d'emblée comme une œuvre fondatrice non seulement en raison de son mode innovant de mise en œuvre, par précontrainte de voussoirs préfabriqués, mais également du fait de la nouvelle esthétique aux lignes tendues qu'elle inaugurait. Comme les principaux ouvrages techniques marquants de la Reconstruction, les ponts en béton précontraint de Freyssinet allaient connaître un profond retentissement sur l'architecture contemporaine : leur principe structurel inspira par exemple directement celui de la basilique souterraine Saint-Pie-X de Lourdes (1954-1958 ; André Le Donné, Pierre Pinsard et Pierre Vago, architectes) ${ }^{20}$.

Mode de mise en œuvre universel, la précontrainte ne fut pas seulement exploitée par Freyssinet pour la construction de ponts mais également pour l'exécution d'édifices ${ }^{21}$. Et si, grâce à la STUP, les établissements Campenon-Bernard s'étaient imposés comme le leader international de la nouvelle technique, nombre d'ingénieurs et d'entreprises s'y étaient ralliés dès l'entre-deux-guerres, en France comme à l'étranger, notamment en Allemagne. Ainsi, par exemple, Henry Lossier (1878-1962) et Nicolas Esquillan mirent-ils au point de nouveaux procédés, le premier pour la reconstruction d'un pont sur la Seine à Villeneuve-SaintGeorges (1948-1950) et le second, pour celle du viaduc de la Voulte (1952-1954), enjambant le Rhône.

La précontrainte, et ses divers procédés, se développa ainsi d'autant plus rapidement que deux importantes organisations se chargèrent de sa diffusion ${ }^{22}$. Dès 1949 fut fondée l'Association française de la précontrainte qui initia, en 1952, la création de la Fédération internationale de la précontrainte. Dès lors, tous les domaines de la construction intégrèrent les principes fondamentaux de la nouvelle technologie, en particulier celui des voiles minces et des couvertures.

Dans ce domaine, Bernard Laffaille (1900-1955) puis René Sarger (1917-1988) jouèrent un rôle prépondérant qui s'illustra notamment avec la conception de la toiture suspendue de Notre-Dame de Royan et la coque ondulée du marché couvert de la cité balnéaire. Laffaille avait pu auparavant mûrir ses conceptions novatrices dans le cadre de la reconstruction de la SNCF qui l'avait sollicité dès octobre 1944 pour élaborer, en collaboration avec ses services techniques, de nouvelles rotondes pour locomotives à vapeur et de vastes halles aménagées pour le " trafic accéléré » des marchandises. Ces projets s'inscrivirent directement à l'origine de ceux qu'il développa à Royan avec les architectes, dans la mesure où ils mettaient en œuvre les techniques d'avant-garde dont l'ingénieur s'était fait l'un des principaux pionniers depuis les années 1920 : voiles minces à courbures, coques plissées et couvertures suspendues et prétendues ${ }^{23}$.

Dans ce domaine des voiles minces, I'ingénieur Nicolas Esquillan (1902-1989), directeur technique des établissements Boussiron, construisit pour un hangar d'aviation sur l'aéroport de Marignane (1942-1951) les plus vastes couvertures du moment en fines coques de béton armé sous la forme d'ondes toriques, raidies de part en part par des tympans verticaux, atteignant la portée record de 100 mètres ${ }^{24}$. C'est à partir de l'expérience acquise à l'occasion de ce remarquable projet que le directeur technique de l'entreprise Boussiron conçut quelques années plus tard les voûtes du CNIT à Paris qui
16. Globalement, l'ingénierie des travaux publics s'avéra en effet plus innovante durant la Reconstruction que celle du bâtiment, malgré les premières expérimentations en matière d'industrialisation du logement. Voir D. Barjot, «Les entreprises du bâtiment et des travaux publics et la Reconstruction (1918-1945), dans B. Vayssière,

Reconstructions et modernisations, op. cit. note 1, p. 234.
17. Citons, entre autres, deux ouvrages emblématiques de la période, conçus par Nicolas Esquillan et réalisés par l'entreprise Boussiron : le viaduc de la Méditerranée (1948-1950), record mondial de portée des ponts-rails à double voie ferrée suspendue et le magnifique viaduc de la Voulte (1952-1954), premier grand pont de chemin de fer français en béton précontraint (cinq travées de 56 mètres) et le plus long du monde sous voie ferrée normale. Voir Bernard Marrey, Nicolas Esquillan, un ingénieur d'entreprise, Paris, Picard, 1992, p. 54-67.

18. Voir notamment Eugène Freyssinet, «Une révolution dans l'art de bâtir : les constructions précontraintes ", Travaux, novembre 1941.

19. Voir José A. Fernandez-Ordonez, Eugène Freyssinet, Barcelone, Grupo $2 \mathrm{C}$ Éditeurs, 1979, p. 173-224. Sur l'invention de la précontrainte et ses premières 
Centre national des industries et des techniques (CNIT) à Paris-La Défense. Vue aérienne à l'achèvement du chantier, 1958-1959. Nicolas Esquillan, directeur technique

des établissements

Boussiron; Robert

Camelot, Bernard

Zehrfuss et Jean de

Mailly, architectes.

DR.

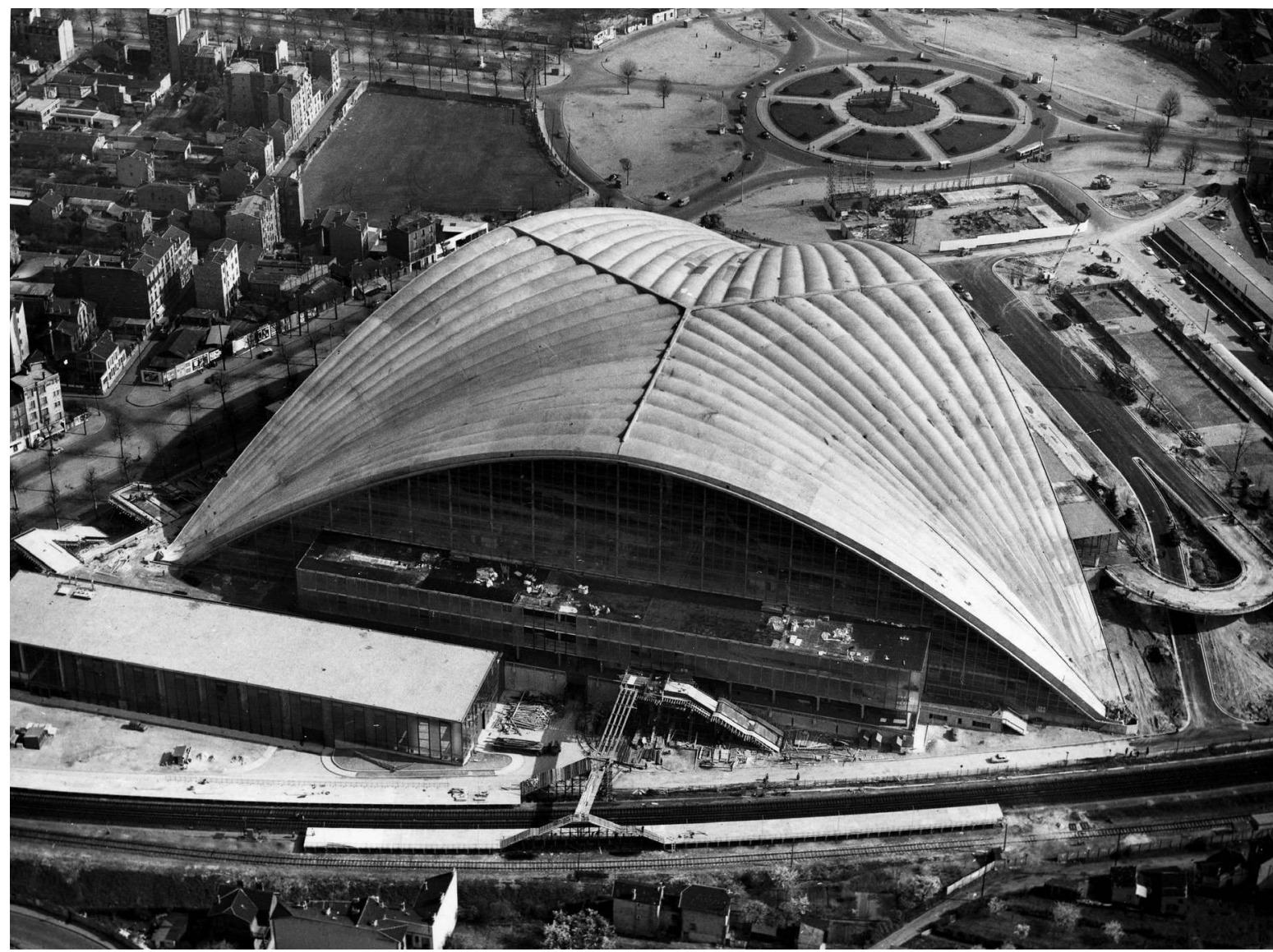

applications, voir en particulier Jupp Grote et Bernard Marrey, Freyssinet, la précontrainte et l'Europe, 1930-1945, Paris, Éditions du Linteau, 2000.

20. Voir à ce propos l'article du directeur des études des entreprises CampenonBernard, J. Chaudesaigues : "La basilique Saint-Pie-X à Lourdes, Travaux, n 291, janvier 1959, p. 9-22, ainsi que le dossier publié dans L'Architecture d'aujourd'hui, n॰81, décembre 1958, p. 46-55.
21. Par exemple: des réservoirs d'eau de 7000 mètres cubes à Orléans en 1948, le phare de Berck en 1950 ; et des ouvrages d'infrastructures considérables en collaboration avec les services des Ponts et Chaussées : la galerie couverte de Rouen de 1800 mètres de longueur sur la rive gauche de la Seine (1948) ou la reconstruction du quai de France dans le port de Cherbourg, achevée en 1951.
22. Sur l'essor et la diffusion de la précontrainte, voir D. Barjot, op. cit. note 2, p. 131-143.

23. Voir Nicolas Nogue, «Le temps de l'ingénieur et le temps de l'architecte ou construction industrialisée et architecture monumentale pendant la Reconstruction » dans Gérard Monnier (dir.), Le Temps de l'œu ure. Approches chronologiques de l'édification des bâtiments, Paris, Publications de la Sorbonne, 2000, p. 13-29. 


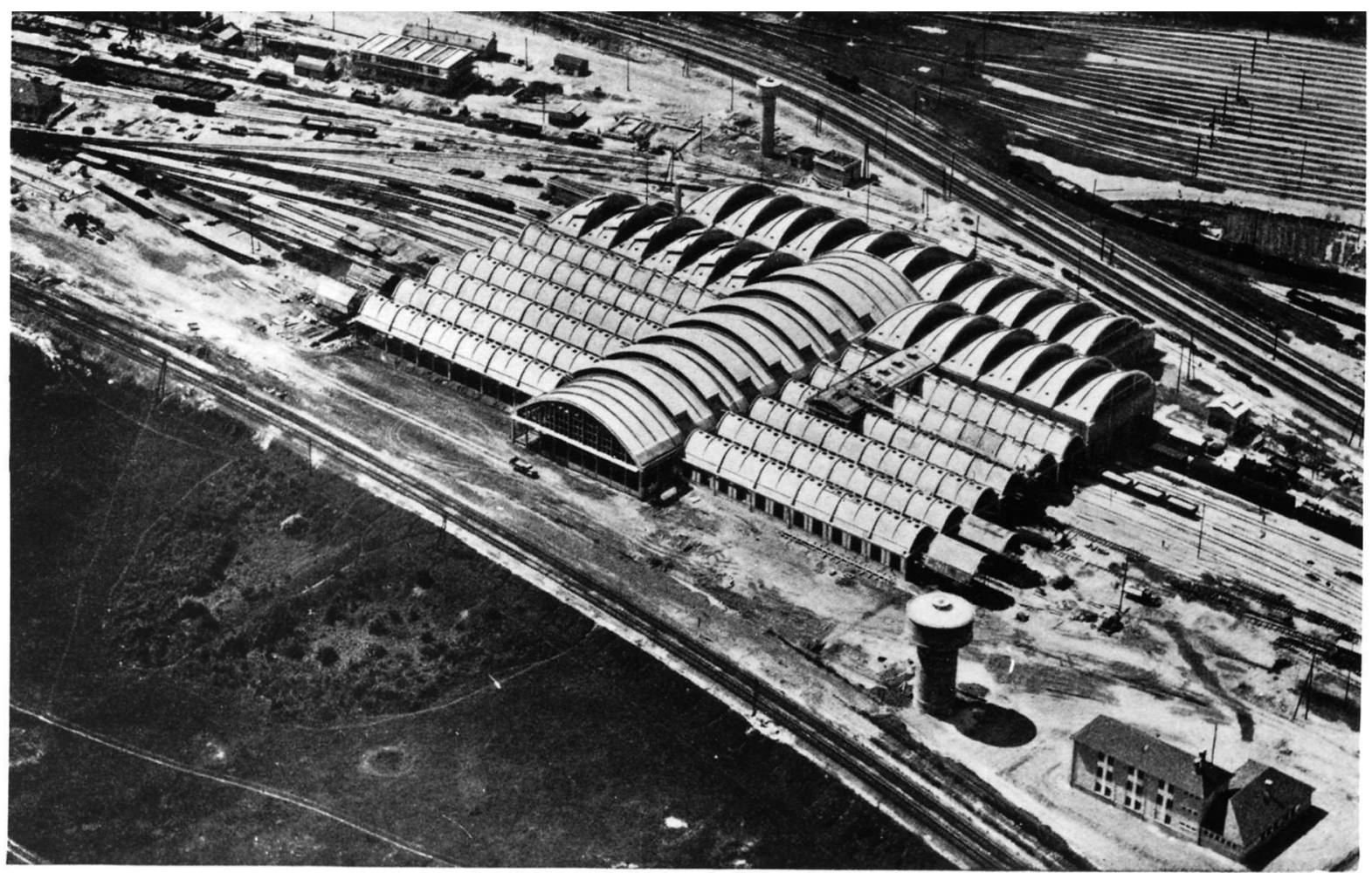

Remise à locomotives à

vapeur SNCF. Dépôt de

Châlons-en-Champagne.

1949. Albert Caquot,

ingénieur-conseil.

Doc. Sciences et Industries. 
exploitaient, sous la configuration d'une double coque, des structures apparentées à celles mises en œuvre à Marignane, mais à une échelle double, puisque elles couvrent un plan triangulaire de 206 mètres de longueur en façade et 240 mètres sous les arêtes de noues.

Parmi les ouvrages emblématiques de la « Reconstruction des ingénieurs ", il convient enfin de mentionner les opérations menées dans le domaine de la production d'électricité, barrages et centrales thermiques, dont I'exécution revenaitnotammentà la Compagnie nationale du Rhône, aux Charbonnages de France et, surtout, à Électricité de France, nouvellement nationalisée. Priorité du plan Monnet, le programme hydroélectrique donna lieu à de nombreux ouvrages hydroélectriques (pas moins de 70 chantiers sont lancés dans les années 1940 et 1950), parmi lesquels figurent des réalisations exceptionnelles où s'illustra André Coyne (1891-1960), spécialiste mondialement reconnu, ancien ingénieur des Ponts et Chaussées qui avait fondé en 1947 son bureau d'études Coyne \& Bellier. Proche de Freyssinet, Coyne employait dès 1928 de nouveaux procédés de précontrainte et développa en particulier la technique des barrages-voûtes à double courbure (permettant des économies de l'ordre de $40 \%$ à $50 \%$ par rapport aux barrages-poids équivalents) ${ }^{25}$.

Pour les aménagements de l'Aigle sur la Dordogne, achevés en 1946, il mit au point une nouvelle typologie d'ouvrage intégrant la centrale électrique dans le corps du barrage, imposant ainsi la construction d'un déversoir "en saut de ski ", selon sa propre expression, configuration qu'il allait employer pour de nombreux autres projets et qui connut un retentissement considé- rable. Ainsi, aussi étonnant que cela puisse paraître, Le Corbusier s'en inspira pour déterminer la forme de la toiture de l'église de Ronchamp non seulement en raison de sa saisissante plasticité mais aussi pour la fonctionnalité de sa forme : I'architecte reprit la coupe de ce barrage pour trouver un galbe qui favorise l'écoulement des eaux du toit de la chapelle ${ }^{26}$.

Il est vrai que par leurs dimensions, les barrages figuraient parmi les plus importantes réalisations du moment nécessitant des installations de chantier hors normes ${ }^{27}$ : le magnifique barrage à voûtes multiples de la Girotte, réalisé en 1948 par l'entreprise EMC sur les plans d'Albert Caquot (1881-1976), élève ses puissants voiles en béton massif à 48 mètres de hauteur pour ménager une ligne de crête de 500 mètres de long. Celui de l'Aigle atteint 95 mètres de hauteur pour une longueur de couronnement de 290 mètres. L'importance des travaux, combinée à la pénurie de main-d'œuvre spécialisée ainsi qu'à l'augmentation des salaires et des charges, avait conduit à une rationalisation de l'organisation des chantiers et surtout à leur mécanisation poussée qui préfiguraient celle des futurs "grands ensembles» de logements. C'est notamment pour l'aménagement des barrages qu'apparaissent les premières grues hydrauliques Poclain et les premiers «bulldozers".

Outre les barrages, les centrales thermiques constituaient le second volet du programme électrique impulsé par le plan Monnet. II devint d'ailleurs rapidement prioritaire en raison de sa rentabilité supérieure sur celui des aménagements hydroélectriques ${ }^{28}$. Dans ce domaine également, les réalisations les plus marquantes furent sans conteste les centrales développées par les
24. Outre la performance des structures, l'opération innove également en raison du mode de mise en œuvre adopté par coulage au sol des couvertures puis levage à l'aide de vérins. Voir Nicolas Esquillan, "Le hangar en béton armé à deux nefs de 101,50 m de portée de l'aéroport de Marignane ", dans Mémoires de la Société des ingénieurs civils de France, 16 avril 1952.
25. Voir D. Barjot, op. cit. note 2.

26. Voir Danièle Pauly, Le Corbusier : La chapelle de Ronchamp, Paris, Fondation Le Corbusier, Bâle, Boston, Berlin, Birkhäuser, 1997, p. 90-92. L'historienne précise : "Si l'on compare cette coupe [du barrage de l'Aigle] à la courbe formée par la déclivité du toit depuis l'angle sud-est jusqu'au côté ouest où l'eau s'écoule par la gargouille, I'analogie formelle est évidente. »
27. Voir Jean-Louis Bordes, Les Barragesréservoirs en France, du milieu du XVIII au début du $x x^{e}$ siècle, Paris, Presses de l'École nationale des ponts et chaussées, 2005. 
Houillères du bassin de Lorraine, entre 1947 et 1953, à Carling et à Grosbliederstroff en raison des réfrigérants qu'elles érigèrent sous forme d'hyperboloïdes de révolution en coque de béton armé, hauts de 85 mètres pour un diamètre au sol de 60 mètres $^{29}$ : avec les voiles en paraboloïdes hyperboliques, cette forme-structure nouvelle allait devenir emblématique de la modernité architecturale des années 1950 et 1960.

Frappés par les nouvelles possibilités architecturales que ces techniques offraient, tant fonctionnelles qu'esthétiques, les architectes les exploitèrent ensuite pour les programmes les plus divers : à Royan, Simon et Morisseau les utilisèrent pour le marché couvert ainsi que Guillaume Gillet pour l'église Notre-Dame, les châteaux d'eau de Belmont et Saint-Pierre, Le Corbusier aussi pour couvrir la salle de l'Assemblée du Parlement de Chandigarh (1951-1961) et dans ses premières esquisses pour l'église de Firminy (1955). C'est encore un hyperboloïde de révolution qui constitue la tour-lanterne de la basilique d'Alger (1956-1962 ; Herbé et Le Couteur, architectes ; Sarger, ingénieur) et dont s'inspire Oscar Niemeyer pour composer, à l'aide de poutres élancées, l'élégante structure de la cathédrale de Brasilia (1959-1970). Le plus souvent, ces édifices empruntèrent aux projets industriels leur nouveau mode économique de mise en œuvre par coffrage grimpant.

\section{Le cas des installations aéronautiques}

Dans le domaine de l'aéronautique, la reconstruction des installations civiles et militaires s'effectue sous la tutelle du ministère des Travaux publics et des Transports dans le cadre du «Plan quinquennal (1946-1951) de remise en état des aérodromes et d'amélioration des aéroports ", établi par René Lemaire, inspecteur général des bases et routes aériennes ${ }^{30}$. En 1945, 450 aérodromes sont en effet détruits ou endommagés et plus de 2 millions de mètres carrés d'installations rasés. À part la reconstruction de l'aérogare du Bourget et l'aménagement d'un nouvel aérodrome à Nice, le plan prévoit principalement une remise en état des aérogares ${ }^{31}$. Pour ce faire, a été fondé, en 1946, le Service technique des bases aériennes (STBA). Dirigé par le corps des ingénieurs des Ponts et Chaussées, le STBA est un bureau d'études à compétence nationale. Il assure des missions centralisées de maîtrise d'ouvrage et de maîtrise d'œuvre en développant notamment une normalisation stricte des projets pour chaque type d'équipement bâti. Cette politique normative préfigure celle qui a été mise en place plus tard dans le domaine des établissements scolaires et du logement collectif.

Sur le plan local, apparaissent conjointement les premières entités administratives et opérationnelles dont I'autorité ne se limite plus uniquement à un seul aérodrome ou aéroport mais s'étend sur un territoire départemental. Dans le Sud-Est de l'Hexagone, l'arrêté du 26 avril 1946 signé par Jules Moch, ministre des Travaux publics et des Transports, fonde le premier Service spécial des bases aériennes (SSBA) dont l'activité couvre l'ensemble des aménagements du département des Bouches-duRhône ${ }^{32}$. En collaboration avec le STBA, le nouveau SSBA dresse le bilan des destructions, établit la cartographie des aérodromes et conçoit les plans de masse des bases aériennes, des aéroports et de leurs installations (bâtiments, hangars, pistes et aires de stationnement).
28. En 1950, les centrales thermiques produisaient déjà $52 \%$ de l'électricité en France.

29. Voir L'Architecture d'aujourd'hui, $\mathrm{n}^{\circ} 27$, décembre 1949, p. 22-23.
30. Denise Brimeur, Vital Ferry, Bernard Pelissier et Marc Suel, Regards sur I'aviation civile. Histoire d'une administration, Paris, Cépaduès Éditions, 1992, p. 57-100.
31. René Lemaire, «Plan d'équipement aéronautique ", Techniques et Architecture, nos 9-12, 1947, p. 453-462.

32. 50 ans au Service Spécial des Bases Aériennes 1946-1996. Histoire du SSBA Sud-Est, brochure éditée par le SSBA Sud-Est, Marseille, 1996. 


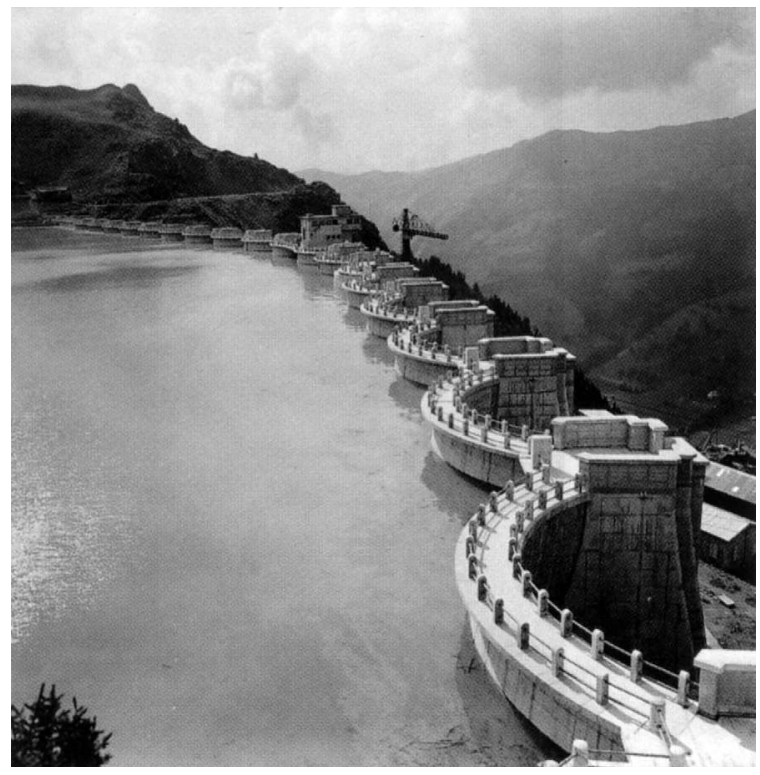

Barrage de La Girotte, 1948-1950. Albert Caquot, ingénieurconseil ; EMC,

entrepreneur ; EDF, maître d'ouvrage. DR.
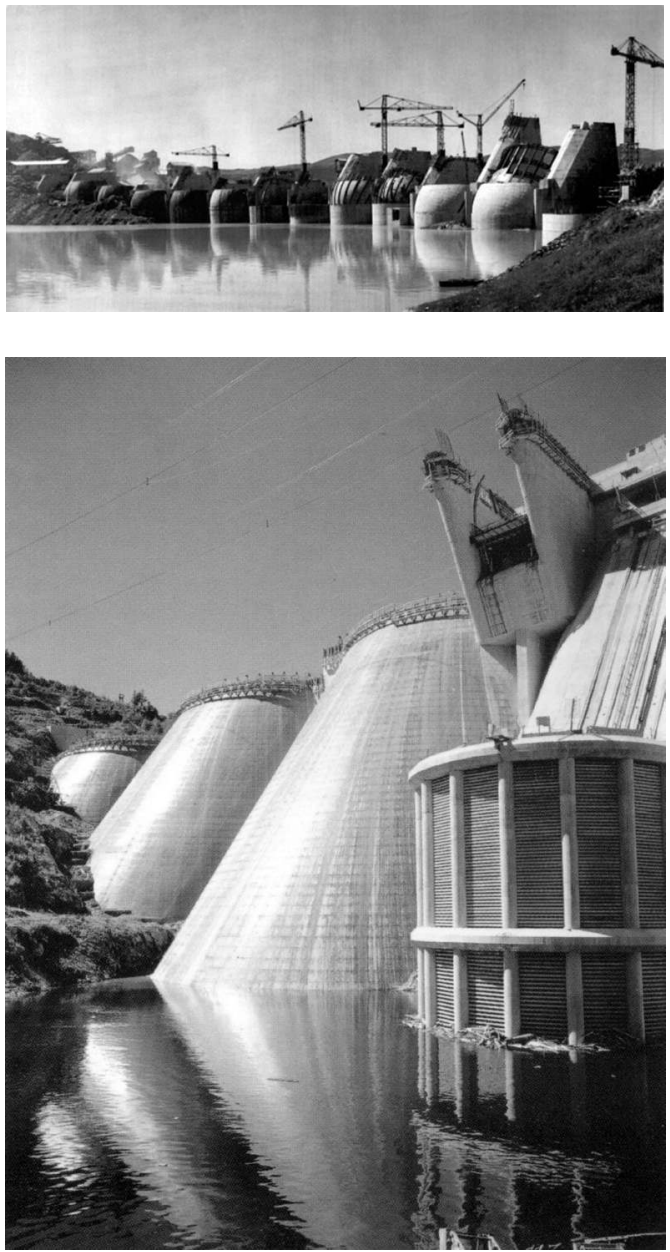
Parmi ses plus importants chantiers figurent l'aéroport principal de Marseille-Marignane, l'aérodrome militaire d'Istres, la base-école de Salon-de-Provence, I'aérodrome militaire d'Aix-Les-Milles et la base aéronavale de Berre $^{33}$. En raison de l'implantation d'industries aéronautiques en bordure des terrains aménagés par le SSBA, ce dernier est également chargé de la reconstruction de leurs usines, en particulier celles de la Société nationale de construction aéronautique du sud-est (SNCASE). Dès sa création, le SSBA des Bouches-du-Rhône est placé sous la direction de l'ingénieur des Ponts et Chaussées Henri Mazen, qui fut par ailleurs chargé de mettre en place le cours sur l'aménagement des bases aériennes à l'École nationale des ponts et chaussées.

Sur le modèle des Bouches-du-Rhône, un second Service spécial des bases aériennes a été créé en Gironde (arrêté du $1^{\text {er }}$ juillet 1946), en raison de la densité et de l'importance des installations aéroportuaires existant autour de Bordeaux. En Île-de-France, la Reconstruction fut gérée différemment. Le 24 octobre 1945, le gouvernement crée par ordonnance l'établissement public Aéroport de Paris (ADP) qui reçoit pour mission de " concevoir et aménager l'ensemble des aérodromes ouverts à la navigation civile dans un rayon de 50 kilomètres autour de la capitale ". Sa direction des Études et Travaux est placée sous à la houlette de Pierre D. Cot, ingénieur en chef des Ponts et Chaussées ${ }^{34}$. Dès 1946, ADP rebâtit en béton armé et à l'identique l'aérogare du Bourget en collaboration avec l'architecte du projet initial, le premier grand prix de Rome Georges Labro. Toute I'organisation des fonctions et circulations de l'ouvrage ont été repensées à cette occasion qui représente alors la seule reconstruction d'envergure d'un projet monumental parmi les installations aéronautiques.

En 1947, l'établissement public termine la construction de l'aérogare provisoire (en bois) et de la première piste bétonnée à l'aéroport d'Orly ${ }^{35}$. La même année, l'architecte et ingénieur des Ponts et Chaussées Henri Vicariot intègre le service d'études et de travaux d'ADP. Avec l'ingénieur des Ponts et Chaussées E. Becker, il mène dès lors la conception des ouvrages majeurs des aérodromes civils d'île-de-France et, surtout, tous les nouveaux édifices de l'aéroport d'Orly, en particulier : la première tour de contrôle, l'aérogare de fret, les bureaux et les hangars dont les ossatures en porte-àfaux, réalisées en poutrelles d'acier tridimensionnelles, représentent les structures métalliques les plus hardies du moment ${ }^{36}$. Parallèlement, Vicariot entame en 1947 la conception de la nouvelle aérogare d'Orly-Sud dont les études ont duré dix années. L'ouvrage est achevé en 1961 en même temps que la nouvelle aérogare de MarseilleMarignane due à Henri Mazen et Fernand Pouillon. Après la reconstruction du Bourget, les deux projets constituent les premiers ouvrages modernes et monumentaux de l'architecture aéronautique d'après-guerre.

\section{L'industrialisation du bâtiment}

Parallèlement à la reconstruction des grandes infrastructures de transport et d'énergie, la contribution des ingénieurs à la reconstruction du pays se manifesta enfin avec éclat dans le domaine de l'industrialisation du bâtiment. L'État, et en particulier le MRU, l'encouragea au point d'en faire I'un des axes majeurs de sa politique technique. Les
33. Henri Mazen, «L'Equipement aéronautique de la région marseillaise », Techniques et Architecture, $n^{\circ}$ 9-12, 1947, p. 488-493.
34. L'armée de l'air a tenu à conserver sa souveraineté sur les terrains militaires, notamment à Villacoublay.

35. Bob Hawkins, Gabriele Lechner et Paul Smith (dir.), Historic Airports: proceedings of the international "L'Europe de l'air " Conferences on Aviation Architecture: Liverpool (1999), Berlin (2000), Paris (2001), Londres, English Heritage, 2005.
36. Voir Techniques et Architecture $n^{\circ} 4$, $17^{\text {e }}$ série, 1958, p. 76 et « Bâtiment du parc central à Orly », Acier Stahl Steel, n० 1, janvier 1956, p. 35-36. 
carences de l'entre-deux-guerres auxquelles s'ajoutaient les destructions du conflit mondial, l'exode rural et la croissance démographique, rendaient en effet urgent de construire plus rapidement et à moindre coût un nombre accru de logements et d'équipements publics (établissements scolaires, sanitaires et sociaux notamment).
L'administration récolta dans un premier temps les fruits de réflexions et d'expérimentations sur la normalisation et la préfabrication conduites au sortir de la Première Guerre mondiale. Elles furent relancées sous Vichy par l'Afnor et le COBTP qui primèrent certains procédés (composants de plancher, de bloc-eau et

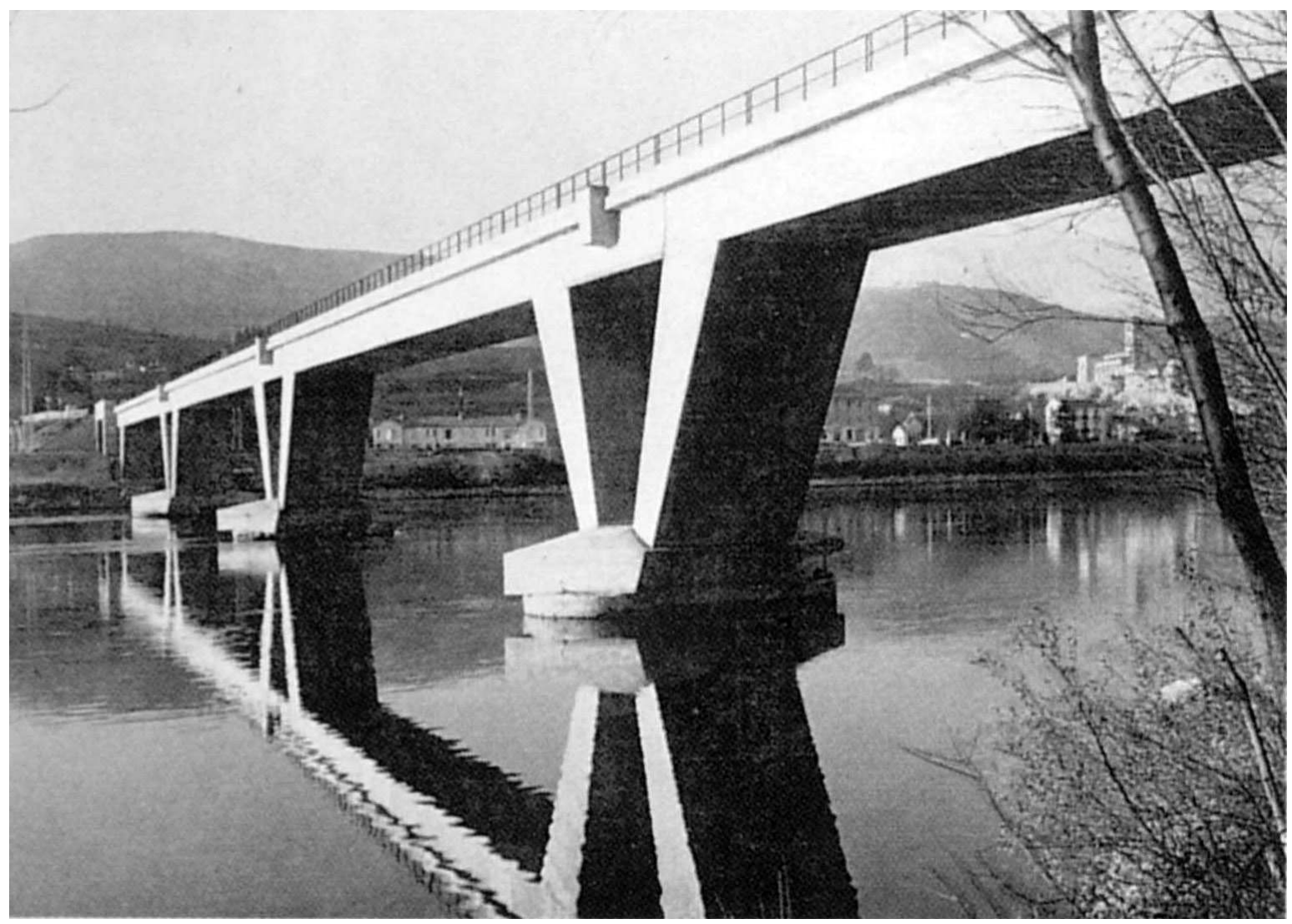

Viaduc de la Voulte (1952-1954).

Nicolas Esquillan, ingénieur; Boussiron, entreprise ; $\mathrm{SNCF}$, maître d'ouvrage. D.R. 
de bloc-croisée...) appliqués au lendemain de la guerre, notamment par l'atelier Perret au Havre et par l'architecte Pol Abraham à Noisy-le-Sec où, dès 1946, un chantier expérimental permitégalementl'exécution, par préfabrication totale, de maisons individuelles. S'appuyant sur ces expériences, le MRU stimula par la suite l'industrialisation par une politique de " chantiers d'expé- rience » ouverts sur concours, de « conception-construction » dirait-on aujourd'hui, qui imposaient la constitution d'équipes intégrant architectes, bureaux d'études et entreprises et qui favorisaient l'expérimentation à grande échelle des normes, matériaux et nouveaux procédés, dans le cadre de programmes de plus en plus importants.

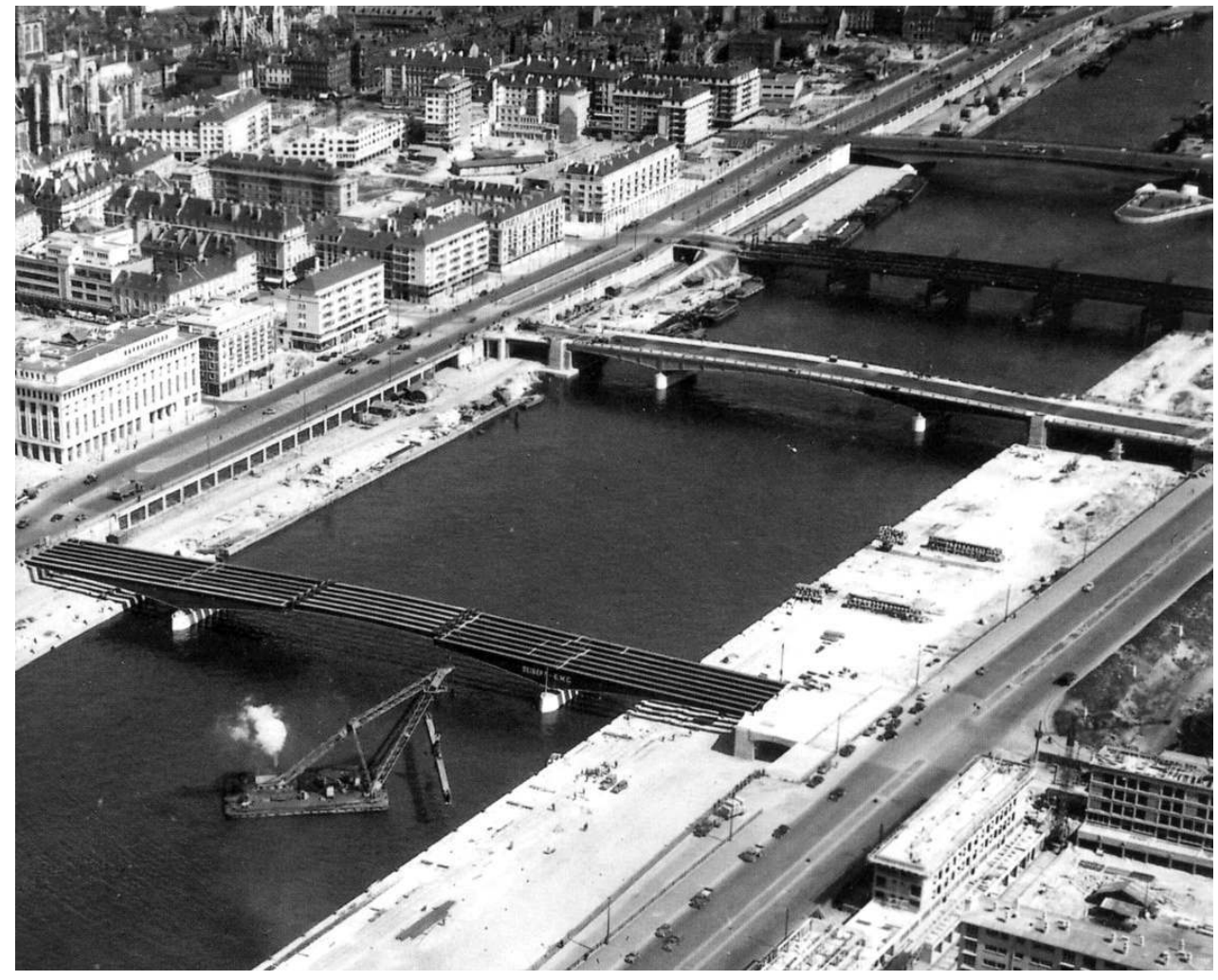

Chantier de reconstruction des berges de la Seine et des ponts à Rouen (Seine-Maritime).

Document Drac Normandie, 1955. 
En 1947, le « concours pour l'édification de maisons nouvelles » porta sur des groupes de 50 logements (en individuel ou en collectif) sur l'ensemble du territoire. En 1949, la même politique est poursuivie pour la réalisation d'ensembles de 200 logements répartis sur quatre sites (Compiègne, Chartres, Creil et Villeneuve-SaintGeorges). Enfin, en 1951, le concours de Strasbourg, remporté par le prix de Rome Eugène Beaudouin associé à l'entreprise Boussiron, marquait avec son programme de 800 logements l'apogée et la fin de ces " chantiers d'expérience " pour entrer dans l'ère du logement de masse et des " grands ensembles ».

Toutefois, malgré ces programmes phares mais ponctuels, l'industrialisation de la mise en œuvre ne touchait encore, en 1950, qu'une part marginale de la construction ${ }^{37}$. Les pouvoirs publics décidèrent alors de la développer considérablement dans le cadre du « secteur industrialisé » visant l'édification de 12000 logements par an. Mise au point et appliquée de 1952 à 1957 par le directeur de la Construction du MRU, l'ingénieur des Ponts et Chaussées Adrien Spinetta, cette nouvelle politique organisa le lancement de grandes opérations, de l'ordre de 1000 logements, favorisant l'emploi de procédés de préfabrication sélectionnés et l'intervention en amont des bureaux d'études et des entreprises pour la conception de projets d'exécution très précis, donc plus économiques ${ }^{38}$.

Le «secteur industrialisé » amplifiait ainsi la politique précédente des chantiers d'expérience en permettant la généralisation de modèles architecturaux et techniques. II intervenait ce faisant sur les structures de production en faveur des BET et des entreprises mais au détriment des architectes dont la créativité s'en trouva brimée. En 1953, Pierre Courant, ministre de la Reconstruction, mettait en place un plan d'ensemble pour le logement économique qui allait dans le même sens, en s'appuyant davantage sur les organismes HLM et en apportant d'indispensables réformes foncières.

Dans le cadre de cette politique publique, les ingénieurs ont occupé une place essentielle et croissante au point de devenir dominante. Qu'ils aient œuvré au sein de bureaux d'études ou d'entreprises, ce sont eux les inventeurs des principales méthodes de préfabrication alors employées dans la construction de logements et dont les procédés Camus, Coignet, Balency \& Schuhl, Barets ou ceux de Jean Prouvé sont devenus emblématiques ${ }^{39}$.

Cela étant, au début de la Reconstruction, les nouveaux procédés étaient généralement appliqués à des projets maîtrisés par les architectes, comme ceux de Camus, à Shape Village à Saint-Germainen-Laye (1951-1952 ; Jean Dubuisson, architecte) ou au Havre. À partir du début des années 1950, en raison de la croissance considérable de la taille des opérations industrialisées et de la nécessité qui en découlait de renforcer en amont les études techniques et économiques, les architectes perdirent progressivement au profit des bureaux d'études et des entreprises une part importante du contrôle de la conception de ces projets dans la mesure où leur formation (et peut-être aussi la structure de leurs agences) n'était pas adaptée aux nouvelles exigences sociales de l'industrialisation du bâtiment ${ }^{40}$.
37. Guy Lambert, Valérie Nègre, «Les chantiers de l'innovation constructive », dans Les Grands Ensembles. Une architecture du $x x^{e}$ siècle, Paris, Dominique Carré Éditeur, 2011, p. 189-206.

38. La première tranche du « secteur industrialisé » fut lancée sur six projets totalisant plus de 7000 logements à Saint-Étienne, Angers, Bron-Parilly, Le Havre, Boulogne-sur-Mer et Pantin (voir Ministère de la Reconstruction et de I'Urbanisme
1944-1954. Une politique du logement, op. cit. note 5, p. 96).

39. À cet égard, il faut souligner le rôle fondamental joué dans ce vaste processus d'innovation par les divers centres de recherche, d'expérimentation et de diffusion de l'information, qu'ils soient publics, comme le CSTB ou le Laboratoire central des ponts et chaussées créé en 1949, ou surtout privés, à l'instar du Centre expérimental de recherche et d'études du
BTP, fondé dès 1933, du Laboratoire des liants hydrauliques et de la céramique, et du Centre d'études du bâtiment et des travaux publics (CEBTP), établi en 1953. Sans que I'on puisse s'appuyer sur des recherches historiques rigoureuses, il ne fait guère de doute que les ingénieurs occupaient la plupart des postes de commande de ces organismes. 
II s'agit également de montrer que cette " reconstruction des ingénieurs " s'opère à la fois sous l'égide d'une économie planifiée mais "économiquement concertée " et dans le cadre de la mise en place d'une politique d'aménagement inédite en France visant « une répartition harmonieuse des hommes et des activités sur le territoire ${ }^{41} »$. À ce titre, les principales interventions des ingénieurs semblent s'être davantage inscrites dans une politique générale d'aménagement territorial où s'articulent de manières plus ou moins cohérentes des actions d'ampleur nationale, décidées au sommet de l'État, et des dynamiques régionales déléguées aux acteurs économiques et politiques locaux, le tout financé par le premier plan, le " plan Monnet » (1947-1951), clairement volontariste voire dirigiste, envisageant comme autant d'occasions propices à une projection en avant, autant d'opportunité de modernisation les reconstructions des grands équipements de transports, de production d'énergie, d'industrie lourde et de l'agriculture à l'aune de l'efficacité économique ${ }^{42}$. En comparaison, la politique de reconstruction urbaine largement confiée aux architectes n'a pas donné lieu à l'élaboration d'une politique urbaine globale, à la mise en place d'éléments de doctrine imposés nationalement, d'autant que le processus de remembrement, de choix des architectes et les décisions en termes de financement des travaux devaient tenir compte des prérogatives locales des Associations syndicales de reconstruction (ASR), défendant les intérêts des propriétaires sinistrés ${ }^{43}$. C'est à leur initiative que les projets de Le Corbusier à La Rochelle puis à Saint-Dié n'ont pu voir le jour. Cette différence majeure dans le processus de prise de décision explique que la reconstruction de la grande majorité des villes françaises par les architectes a essentiellement représenté un chantier de rattrapage ou de mise à niveau qui ne laissa place à aucune géographie volontaire. Elle n'a pu être l'occasion ni de repenser l'édification des cités détruites comme un projet ouvert de modernisation globale ni de repenser la rénovation de l'armature urbaine nationale comme ce fut le cas, sous la houlette des ingénieurs, dans le domaine des équipements et de l'industrie.
40. Voir Christel Palant-Frapier, "L'émergence des bureaux d'études techniques en France autour de 1950 ", Entreprises et Histoire, $n^{\circ} 71$, "Les entreprises françaises d'ingénierie face à la compétition internationale », numéro dirigé par Dominique Barjot, 2013, p. 100-110.
41. Jean-François Gravier, Paris et le désert français. Décentralisation, équipement, population, Paris, Le Portulan, 1947.

42. M. Desportes, A. Picon, op. cit. note 3.

43. Voir D. Voldman, op. cit. note 1, p. 194. 